\title{
Short-Term Semantic Consensus: Towards Agile Ontology Specification for Collaborative Networks
}

\author{
Carla Pereira ${ }^{1,2}$, Cristóvão Sousa ${ }^{1,2}$, and António Lucas Soares ${ }^{1,3}$ \\ ${ }^{1}$ Instituto de Engenharia de Sistemas e Computadores do Porto \\ ${ }^{2}$ Escola Superior de Tecnologia e Gestão de Felgueiras (IPP) \\ ${ }^{3}$ Faculdade de Engenharia da Universidade do Porto \\ csp@inescporto.pt, cpsousa@inescporto.pt, als@fe.up.pt
}

\begin{abstract}
This paper presents our method to support the collaborative conceptualisation process focusing our strategy for building consensus in the context of collaborative networks. This new strategy comes from the application of the results and recommendations obtained in an experimental evaluation performed in the scope of a large European project in the area of industrial engineering. The usage of our strategy and the collaborative platform supporting semantic consensus building in the scope of the European research project H-Know is described.
\end{abstract}

Keywords: Conceptualization, social construction of meaning, ontology development, collaborative networks, conceptual blending, consensus building.

\section{Introduction}

Although there is an increasing number of semantic tools and resources available for the companies to use in everyday business activities, problems in establishing a common conceptualisation of a given reality arise in two flavours: (i) notwithstanding the evolution of semantic technologies, it is virtually impossible to establish a priori comprehensive and complete semantic artefacts that account for all the possible variations in business situations and contexts (which are more and more dynamic); (ii) in spite of all the standardization efforts, there is a kind of "social resistance" in accepting semantically oriented standards (viewed as "grand narratives" of a domain). A good example of this is the construction industry, where an enormous effort and money has been spent in standard terminologies, vocabularies, thesaurus, ontologies, etc. with results well behind the expected (Silva et al., 2006).

Cahier et al., 2005 argue about the role of a "socio-semantic web": "we need to go beyond the approaches that provide a high level of 'automation of the meaning'; instead, we need to address situations where human beings are highly required to stay in the process, interacting during the whole life-cycle of applications, for cognitive and cooperative reasons".

Even though the most used definition of ontology (Gruber, 1993) "An ontology is a formal, explicit specification of a shared conceptualisation", underlines the collaborative construction of conceptualisations in the scientific context, we agree that: "While different degrees of formalizations have been well investigated and are now found in various ontology-based technologies, the notion of a shared conceptualisation is neither 
well-explored, nor well-understood, nor well-supported by most ontology engineering tools" (Staab, 2008). Current knowledge about the early phases of ontology construction is insufficient to support methods and techniques for a collaborative construction of a conceptualisation (Pereira and Soares, 2008). The conceptualisation phase is of utmost importance for the success of the ontology. But it is in this phase that a social presence is needed as it requires an actor to reliably predict how other members of the community will interpret the conceptual representation based on its limited description. By incorporating the notion of semantics into the information architecture, we thus transform the users of the system themselves into a critical part of the design. Our view is that ontology engineering needs a "socio-cognitive turn" in order to generate tools that are really effective in copying the complex, unstructured, and highly situational contexts that characterize a great deal of information and knowledge sharing in businesses collaboration. This line of research is therefore directed towards the application of cognitive semantic results in the creation of artefacts acting as socio-technical devices supporting the view that meaning socially constructed through collaboration and negotiation. The first line of this research work deals with the application and extension of the Conceptual Blending Theory (CBT) (Fauconnier and Turner, 1998) to the realm of collaborative semantic tools. The practical application of our approach is to support the co-construction of semantic artefacts by groups of social actors placed in organizational contexts interacting towards a set of common objectives. Simple examples of these artefacts are the creation of a common taxonomy (or ontology) for classifying and retrieving content from an inter-organizational portal, the creation of specific terminological accounts to serve as conceptual references in project tasks, or the specification of ontologies for systems interoperability.

In this paper some increments in our method to support the collaborative conceptualisation process are presented. These increments are consequence of the application of the results and recommendations obtained in an experimental evaluation already executed in the scope of a large European project in the area of industrial engineering (described in (Pereira et al., 2009)). The main contribution reported in this paper is the new approach to consensus building during a collaborative conceptualization process within the context of collaborative networks. The usage of this method in the scope of the European research project $\mathrm{H}-\mathrm{Know}^{1}$ is also explained.

\section{A Method to Support the Conceptualisation Process}

Our proposal to support a collaborative process of conceptualisation is grounded on cognitive semantics, specifically on the Conceptual Blending Theory (CBT) (Fauconnier and Turner, 1998). CBT representation gives rise to complex networks by linking two (or more) input spaces by means of a generic space. The generic space provides information that is abstract enough to be common to all the input spaces. Elements in the generic space are mapped onto counterparts in each of the input spaces, which motivate the identification of cross-space counterparts in the input spaces. A further

\footnotetext{
${ }^{1} \mathrm{H}-\mathrm{KNOW}$ is an European research project in the area of building rehabilitation, restoration and maintenance, particularly in the cultural heritage domain. The project objective is to develop an ICT solution, to support SME's collaborative networks in integrating collaboration, knowledge and learning in the RR\&M field (http://h-know.eu).
} 
space in this model of integration network is the blended space or blend. This is the space that contains a new or emergent structure: information that is not contained in both the inputs. The blend takes elements from both inputs, but goes further on providing additional structure that distinguishes the blend from either of its inputs. In CBT, there are three component processes that produce an emergent structure (Fauconnier and Turner, 1998): (1) composition; (2) completion; and (3) elaboration. Due to the lack of space and scope of this document, we advise interested readers to obtain more information about CBT in the following sources (Fauconnier and Turner, 1998) (Evans and Green, 2006) and (Pereira and Soares, 2008), whereas now we'll continue giving a brief description of our method.

The following is assumed as the initial state: (1) a collaborative network has been formed and its goals and mission are defined and understood by all members (that we call "strategic frame"); (2) a common ontology with certain goals and to be used in a given time-frame has to be developed; (3) each organization has a representative in a "network team" in charge of developing the ontology; the common conceptualisation regarding given domains, processes and tasks, is the first important collective task to undertake by this team; (4) a common conceptualisation is to be collaboratively created through explanation, discussion and negotiation. The proposed method establishes the following steps (see figure 1): (1) each organization has assigned one or more input spaces (only one input space per organization is considered here, for simplicity); (2) each organization represents its conceptualisation proposal through the input space; simultaneously, the organization shares the information and other knowledge sources (e.g., URLs, documents and other contents) which allow the correct understanding of the conceptualisation proposal; no specific knowledge representation technique is proposed, but it is important that it has a graphical nature (in the case study we are using concept maps (Canas et al., 2004) (Eskridge et al., 2006)); (3) by some manual or automated (or something in between) process, a generic conceptualisation is generated (generic space); the common conceptual structure in the generic space should be broad enough to be accepted by all the team members with minimum negotiation; (4) considering the "counterpart" elements (concepts of the input spaces subsumed by concepts of the generic space), the process of creating the blend space is started using selective projection; based on the input spaces, strategic frame, generic space and documentation available in the input spaces (called background information), the blend is "run" to obtain new conceptualisation proposals; (5) new conceptual structures proposed in the blend space are object of negotiation; the concepts for which consensus exists are represented in ("copied" to) the generic space; situations that justify "backward projection" to the input spaces and their modification are analyzed (this analysis will be performed by the users, after obtaining consensus) then the emergent blend structure is validated (confirm or eliminate new concepts that raise in the blend); (6) if input spaces modification takes place, the method should resume at step 4; however the creation of a new blend space, is not necessary; (7) when all participants manifest their agreement with the conceptualisation represented in the generic space, the method instance is finished.

Summarizing, at the end of the process the generic space contains the collective conceptualization. The blend was used during the negotiation process with the goal to improve, enrich and mainly help obtaining consensus (proposing new concepts, modifying, improving or eliminating concepts). 


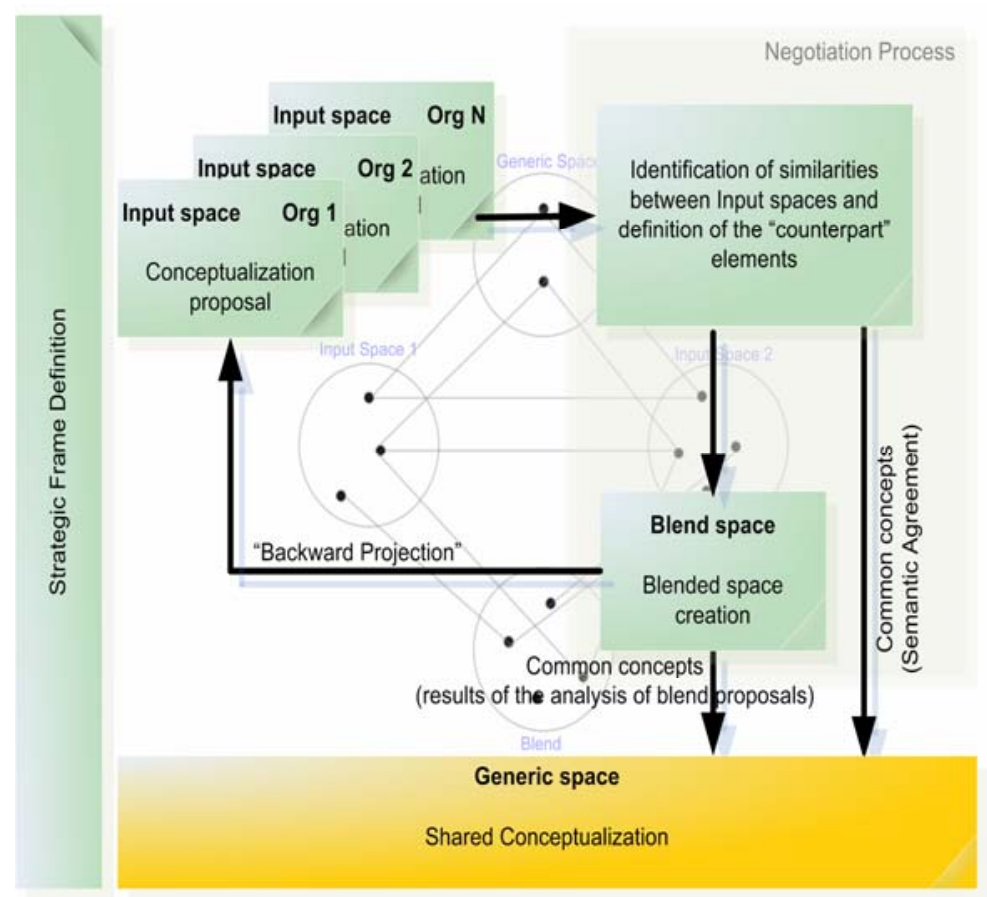

Fig. 1. Method to support collaborative conceptualization process

This method may also be used by each organization to support the creation of its own input space, which can result in the presence of multiple "blendings". It is important to reinforce that in this collaborative process, the validation/agreement achievement requires that each organization indexes to its input space, the sources of information which lead to the input spaces creation and justify the proposal content and structure. Whenever an organization introduces a new concept or association in their input space, it becomes a target for negotiation. At this moment the negotiation process is started. The network team evaluates the new entrance running the steps (3), (4), and (5). To ensure a successful negotiation process, which lead to a shared conceptualisation (accepted by all partners), is also very important to define an approach for obtaining consensus. In the next section we present a strategy for building consensus in the context of collaborative networks. There are some good practices that should be applied, but that need to be adjusted for each case.

\section{Reaching Agreement during the Conceptualisation Process}

We present below a strategy for building consensus in the context of collaborative networks, aligned with the fact that the process of building consensus is regarded collaborative right from the design, once the process design itself should involve all participants (Suskind, 1999). Before developing a specific consensus strategy, the following aspects should be considered: (1) the context in which the process will 
occur, that means that the main intervenient should be aware about the scope of the domain, otherwise some background information should be read; (2) to design the process, it is important to understand the organizational culture of each group of stakeholders. Organizational culture also influences the degree of formality required; (3) when several organizations are involved and their organizational cultures differ, it is important to recognize the specific needs of each group to avoid misunderstandings; (4) the language is also a factor to be considered when different cultural groups are represented; and (5) social and cultural factors. Participants must choose one approach or a combination of the following approaches (see (Susskind et al., 1999)): (i) conventional problem-solving approach; (ii) working with a single-text document; and (iii) taking a visioning approach. Figure 2 shows the phases for the process of building consensus in the context of collaborative networks. Our approach to reach a semantic agreement for the collective conceptualization is thus based on consensus building techniques and involves, besides the negotiation itself, a preparation and an evaluation phases (see figure 2 for the detailed steps).

For the preparation phases, the creation of the team and the governance model of the collaboration are the main tasks. Of particular importance are the selection of the type of participation, the mediation approach, the establishment of rules and responsibilities and the plan elaboration. As for the evaluation phase the most important aspect is to systematise and register the lessons learnt during the process.

In the consensus building phase, must be used a combination of the following two approaches to drive the process, "working with a single-text document" and "taking a visioning approach". The single-text approach involves introducing a working draft of an agreement early in the process for parties to discuss and revise. This approach provides a clear structure for discussions and a focal point to identify areas of agreement and disagreement. A subgroup of participants works to draft a preliminary proposal. This preliminary proposal is considered the best way to focus a consensus building dialogue. This proposal will be presented using concept maps building with CMapTools (http://cmap.ihmc.us/conceptmap.html) (this preliminary proposal is presented in the generic space, see figure 1).

\begin{tabular}{|c|c|c|}
\hline \multicolumn{3}{|c|}{ Semantic agreement building approach - collaborative conceptualisation process } \\
\hline Preparation & Consensus building & $\begin{array}{l}\text { Evaluation } \\
\text { and } \\
\text { lessons learnt }\end{array}$ \\
\hline $\begin{array}{l}\text { - Select the mediator / responsible for the process } \\
\text { - Identify the participants relevant to the process } \\
\text { - Identify a technical resource to ensure the } \\
\text { solution viability } \\
\text { - Prepare the team to participate more productively } \\
\text { - Define the responsibilities of the mediator } \\
\text { - Establish the participation rules } \\
\text { - Define the agenda } \\
\text { - Specify the options for communication }\end{array}$ & $\begin{array}{l}\text { - Using a combination of the approaches } \\
\text { "Working with a single-text document" and } \\
\text { "Taking a visioning approach" } \\
\text { - Keep a record of all items discussed } \\
\text { - Keep all stakeholders informed about the } \\
\text { group progress } \\
\text { - Modify the agenda and procedural rules } \\
\text { (if needed) }\end{array}$ & $\begin{array}{l}\text { - Approval by all involved } \\
\text { - Evaluation of strengths and weaknesses } \\
\text { of the process }\end{array}$ \\
\hline
\end{tabular}

Fig. 2. Consensus building approach 
Brainstorming will be used to expand and improve the preliminary proposal and others proposals that arise during consensus building dialogue (presented in the input spaces of each organisation) as well as to encourage creative thinking. The exploration of several proposals should be accomplished using questions of the type "what if" and performing the steps 3, 4 and 5 of the method to support the collaborative conceptualisation process. The "taking a visioning approach" intend to focus participants' attention toward the future in the course of identifying options and seeking agreements. Conduct the process according to the following questions: What do you have?, What do we want? And "How do we get there? These questions allow the semantic team evaluates the strengths and weaknesses of the current conceptualisation proposal and to maintain the process more creative. To support the dissemination of information, a content management system should be used, that allows to notify the participants in the process, to distribute materials to participants during the process, allow the information sharing, keep all stakeholders updated about the group progress and keep a record of all items discussed. A set of documents such as: meeting minutes, agendas, schedules, working documents and background resources, are taken as important to share. If necessary, at this stage, the agenda and procedural rules can be modified.

\section{A Collaborative Platform to Support Semantic Consensus Building}

A part of our research work is dedicated to design of ITC tools supporting collaborative processes for the development of semantic artefacts according to a socio-semantic stance. For supporting the proposed CBT based methodology, two modules are considered: one supporting the semi-automated construction of the conceptual integration spaces (semBlend) and the other supporting negotiation and consensus reaching (semCons). Our strategy is to develop the semantic artefacts on top of existing collaboration and information management platforms, specifically content management systems (CMS) and wiki systems. In the case of the semCons module, the same platform that was implementing the $\mathrm{H}$-Know project portal was used to implement the module: the Plone CMS. The development involved developing an information structure based on a message board and a workflow implementing the consensus reaching procedure (see figures 3 and 4).

Some remarks about this process follow:

Semantic team creation: (i) There are two different roles that the participants can assume (see figure 3): partner (any member of the project may take this role) and reviewer (each partner organization define a representative who will assume the role of reviewer; (ii) the representatives are part of the group responsible for making decisions; (iii) when the specificity of the conceptualisation increases the need to create different work groups will be evaluated again; (iv) a set of background information as a base to develop skills and the necessary knowledge required to the team was made available in the project portal. 
Planning, responsibilities and roles: (i) The mediator is responsible for ensuring the proper participation of all and managing the conceptualisation; (ii) those who assumed the partner role, are expected to submit proposals and consult, periodically, the state of the process; for the participants engaged as reviewer, it is expected to review, periodically, the proposals submitted by partners and approve, reject or suggest changes. If the proposal is rejected or any change is proposed, the decision must be justified. Only the participants that propose the concept and the reviewers are able to see the candidate concepts. All participants can see the approved concepts. The "reviewers" are electronically notified when a new concept is proposed. A concept is declared as approved whenever at least $80 \%$ of the reviewers vote in favour. However, the ideal is that the concept is approved by unanimity. The reviewers can also propose some changes or clarifications of the concept meaning; in this case the concept is retracted. If a concept is rejected, it can be proposed for change or be rejected definitely. A partner can also dispose a concept. If the reviewers think that a concept is not useful or is no longer part of the common project language, its state would be archived. The replaced state means that the concept was being phased out and was being replaced by a newer concept. The new concept means the same thing as the original but is considered as more appropriated; (iv) the plan should not be too detailed but enough to stress the most important points, dates, etc.

Consensus building: The consensus workflow "execution"; a variety of techniques can be used although in this project we were limited (by the time available) to use the online message board as the main tool.

Evaluation and lessons learnt: Before closing the process, the final version of the conceptualization should circulate for all participants. At this moment should be organized a workshop to divulge the final result to all partners. The evaluation of strengths and weaknesses of the process will be performed based on experience obtained through our participation in the process and carrying out an online questionnaire to all participants.

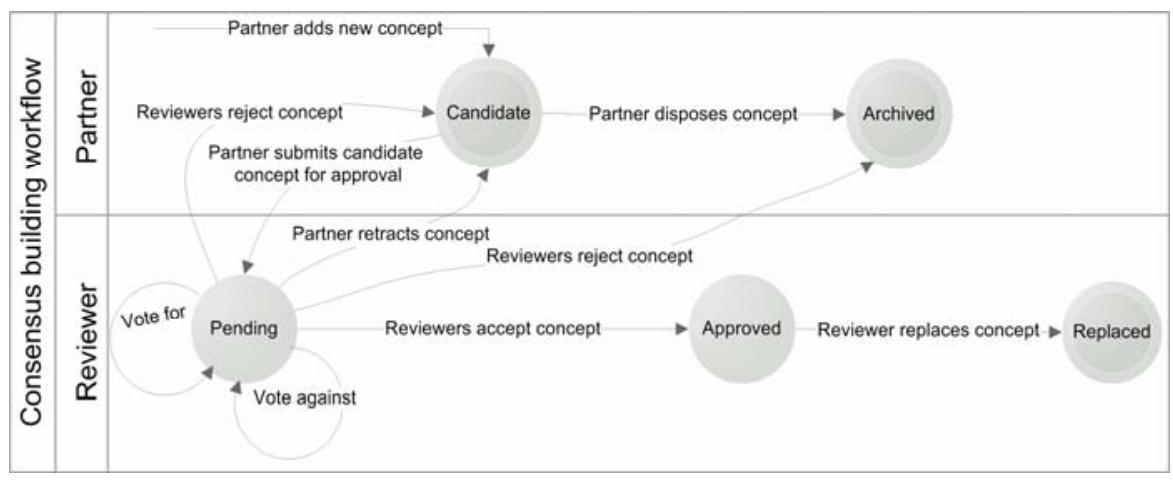

Fig. 3. Consensus building workflow 


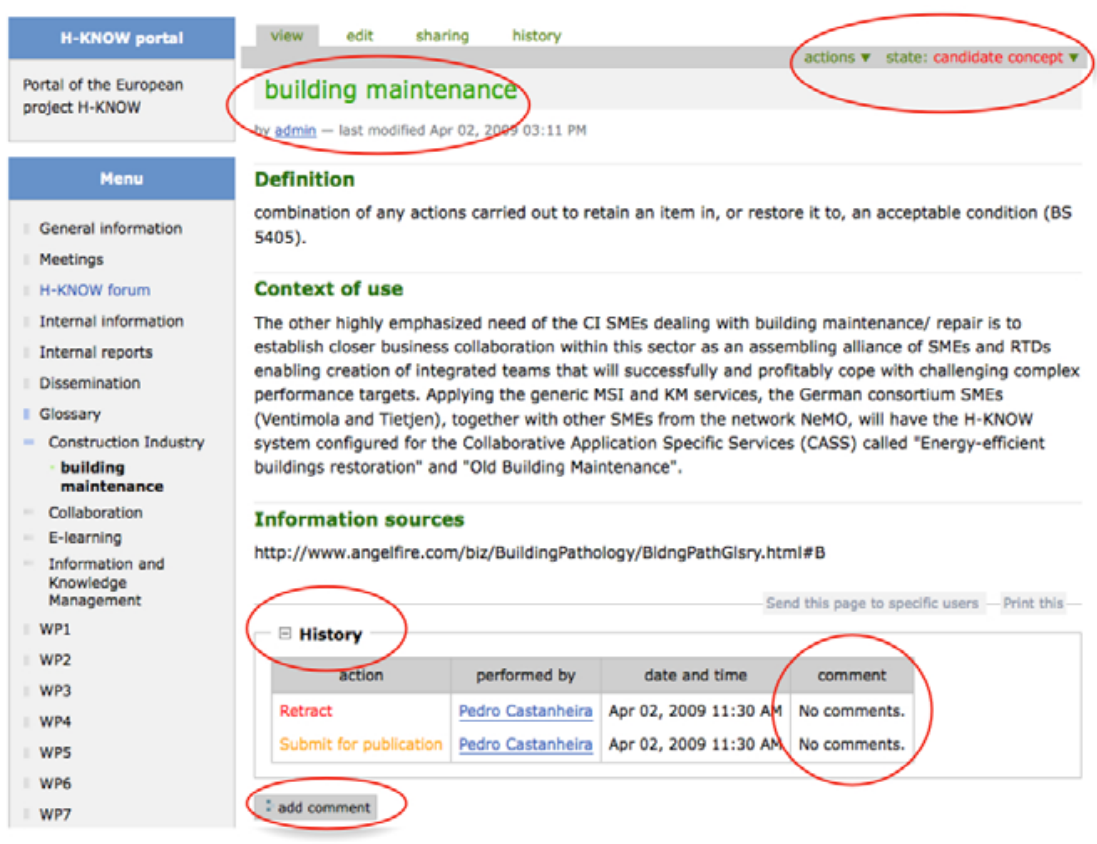

Fig. 4. Example of a concept page in Plone

\section{Conclusions and Further Work}

The approach described in this paper proposes a shift in the process of creation of semantic artefacts from a "semantic artefact engineering" perspective to a "actorartefact co-evolution" one. Socio-semantics is the scientific umbrella to this approach, which is also inspired in cognitive semantics and social networking theories. A set of detailed case studies is under way, whereas action research was adopted as the privileged way to generate knowledge in socio-technical settings. In our process, is already possible to visualize the concept maps inside Plone canvas, which provides an excellent overview about the state of the conceptualisation process. Despite of being a static feature, a graphical representation is a great input for decision support on concepts. Yet, much more benefits can be withdrawn from the CmapTools versatility in knowledge representation of a shared conceptualisation. Concept mapping is a suitable mechanism to emerge that mental framework, which can be used to set up a content management system in a collaborative network environment. On pursuit of this believe, our research and work is targeted to join this two worlds (Concept Mapping and Collaborative Content Management Platforms), in a dynamic, flexible and collaborative environment, according to the architecture depicted in Figure 5. 


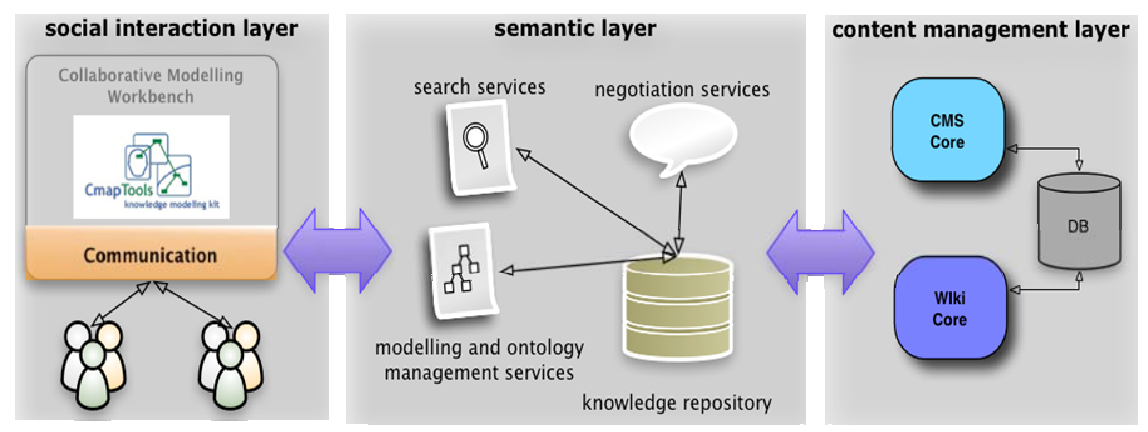

Fig. 5. Dynamic Collaborative Environment Architecture

Regarding the tools development part of our research, Service-Oriented Knowledge Management System is the objective of our future work. According to the Figure 5, the central mechanism of the architecture is the knowledge repository which provides an online location where the semantic team could locate, ideas, documents, tips, etc., from other members. This autonomous data access would make use of common terms representing organizational data. Semantic layer would be powered by a seta set of available services provided by semBlend and semCons modules on supporting to the CBT based methodology. The semantic layer is surrounded by other two layers, the Content management Layer and the Social Interaction Layer. The first, would give support, at the structuring information level, to the artefacts developed on the semantic level. At the Social Interaction Layer, we would have a collaborative workbench, making use of CmapTools, allowing the team to interpret a domain, create models about it and attach meaning via specification of semantic conceptual structures.

\section{References}

1. Cahier, J.-P., Zaher, L., Leboeuf, J.-P., Guittard, C.: Experimentation of a socially constructed "Topic Map" by the OSS community. In: Proceedings of the IJCAI 2005 workshop on KMOM, Edimbourg (2005)

2. Camarinha-Matos, L.: Collaborative networks in industry - Trends and foundations. In: Proc. of 3rd International CIRP Conference in Digital Enterprise Technology. Springer, Heidelberg (2006)

3. Canas, A., Hill, G., Carff, R., Suri, N., Lott, J., Eskridge, T.: T. Cmaptools: A knowledge modeling and sharing environment. Concept Maps: Theory, Methodology, Technology. In: Cañas, A.J., Novak, J.D. (eds.) Proc. of the first Int. Conference on Concept Mapping, Pamplona, Spain, vol. I, pp. 125-133 (2004)

4. Eskridge, T., Hayes, P., Hoffman, R., Warren, M.: Formalizing the informal: a confluence of concept mapping and the semantic web. In: Cañas, A.J., Novak, J.D. (eds.) Proc. of the Second Int. Conference on Concept Mapping, Costa Rica (2006)

5. Evans, V., Green, M.: Cognitive Linguistics: an introduction. Edinburgh University Press (2006) 
6. Fauconnier, G., Turner, M.: Conceptual Integration Networks. Published in Cognitive Science 22(2), 133-187 (1998)

7. Gruber, T.: A Translation Approach to Portable Ontology Specifications. Knowledge Acquisition 5(2), 199-221 (1993)

8. Pereira, C., Soares, A.: Ontology development in collaborative networks as a process of social construction of meaning. In: On the Move to Meaningful Internet Systems: OTM 2008 Workshops. LNCS. Springer, Heidelberg (2008)

9. Pereira, C., Sousa, C., Soares, A.: A socio-semantic approach to the conceptualisation of domains, processes and tasks in large projects. In: 11th International Conference on Enterprise Information Systems, May 6-10 (2009)

10. Silva, M., Soares, A., Simões, D.: Selecting and Structuring Semantic Resources to Support SME'S Knowledge Communities. In: INCOM 2006, 12th IFAC Symposium on Information Control Problems in Manufacturing, St. Etienne, France (2006)

11. Staab, S.: On understanding the collaborative construction of conceptualisations. International and Interdisciplinary Conference Processing Text-Technological Resources at the Center for Interdisciplinary Research ( $\mathrm{ZiF}$ - Zentrum für interdisziplinäre Forschung), March 13-15, Bielefeld University) (2008)

12. Susskind, L., Mckearnan, S., Thomas-Larmer, J.: The Consensus Building Handbook: A comprehensive Guide to Reaching Agreement. The Consensus Building Institute. Sage Publications, Thousand Oaks (1999) 